\title{
Neurodevelopmental Disorders and Autism in Valproic Acid Animal Models
}

\section{Sidra Shafique}

Department of Biomedical and Molecular Sciences, Queen's University, Kingston, Ontario, Canada

\section{Abstract}

Xenobiotics and environmental toxicants cause the perturbations during embryogenesis and Received: August 01,2018 fetal development resulting in congenital malformations. The most vulnerable is the neural tube Accepted: December 10, 2018 development due to the critical window period after the conception of the closure, cellular proliferation Published: December 12, 2018 and differentiation. Neurodevelopmental disorders are multifactorial conditions encompassing wide categories and broad classification. Structural defects reveal as overt anatomical defects while the functional defects become apparent in the postnatal period of brain growth. Autism spectrum disorder [ASD] is an example of functional neurodevelopmental defect. The emphasis in this review is on the fact that neural tube defects are both structural and functional caused by the same teratogens such as valproic acid [VPA]. Valproic acid [VPA] has been discussed as a model teratogen for the development of neural tube defects and autism spectrum disorder [ASD].

\section{Introduction}

Human pregnancy is complicated and even more complicated is finding the ways to study the structural and functional effects of teratogens. Out of all the phases of embryonic and fetal development the first trimester is at the most risk to the developmental complications resulting in birth defects. Nervous system development starts and even gets completed by the time woman is even aware of being pregnant. Therefore, most of the preventive measures need to be addressed either in the perinatal period or to all the reproductive age women at risk of having vulnerable pregnancies. The structural defects are overt and get obvious attention for the management but in fact, the functional defects need more consideration due to their lifelong impacts. There are three modulators of teratogenesis, the susceptibility of species, susceptibility at the time or stage of exposure and the dose of exposure [1]. VPA is an example of a widely prescribed anti-epileptic drug with high teratogenic potential. The VPA-exposed mouse and rat animal models are used to investigate the mechanism and treatment of neural tube defect because VPA results in the development of NTDs and ASD. Understanding the mechanism of action and the pathologies created by VPA would be a gateway to know the pathology, prevention, and treatment landscapes for ASD-like conditions. This review is focused on the structural and functional neurodevelopmental defects with emphasis on valproic acid [VPA] and autism spectrum disorder [ASD].

\section{Neural Tube Development, Defects and Neurodevelopmental Disorders}

Neural tube defects [NTDs] are widespread over the globe with high prevalence in the developing countries. Even after folate fortification, the spina bifida is still $0.05 \%$ of the US live births [2]. Global statistics indicate that NTDs were responsible for about 71,000 deaths in 2010 [3]. US Environmental Protection Agency [EPA] regularly updates the information related to the children's environmental health on America's Children and the Environment [4]. The identified ACE indicators include, under the main group of neurodevelopmental disorders, ADHD, learning disabilities, autism and intellectual disability [4]. Neurodevelopmental disorders are multifactorial that may be genetic or environmental. The environmental causes range from exposure to alcohol to the therapeutics such as valproic acid and infections as of the Zika virus being the cause of microcephaly [5].
The critical period of pregnancy that involves the neural tube development and closure is the first four weeks while most of the women still do not realize that they have conceived a pregnancy due to multiple reasons as of irregularities in the menstrual cycle, failed contraception or even naturally. Prevention, if to be effective, may help during this time of embryogenesis only [6]. The neural tube is formed from the ectoderm of the three-layer embryo. The thickened ectoderm makes neural folds or ridges on the margins of this neural plate, destined by well-articulated gene-regulation, fuse in the midline with a hollow space inside the tube-like structure. The process of fusion, cellular migration, differentiation depends on apoptotic cell death and cell proliferation at the same time [7].

Neural tube defects are primarily structural and functional. The group of structural defects during the neural tube closure, while the embryo is passing through the gastrulation stage, are known as neural tube defects. These are the visible or overt or physical problems that can be seen in the areas of the brain or spinal cord primarily and include the skull and vertebral column defects as secondary extensions, therefore known as structural defects. Examples may include anencephaly and spina bifida [2]. On the other hand, the functional defects in the neurological system which are not overt at birth but are actually the result of the prenatal insult at the same time of neural tube development may include autism spectrum disorders [ASD], developmental delays and learning disabilities [4].

Mostly, the teratogens result through epigenetic changes, dysregulation of developmental genes, and apoptosis through the disturbed cell signaling pathways. Whether structural or functional perturbations, the teratogens are involved at the cellular and molecular levels. Research in the developmental toxicology focuses to investigate how the teratogen causes the abnormalities at the cellular and molecular level causing the functional or structural

"Corresponding Author: Dr. Sidra Shafique, Department of Biomedical and Molecular Sciences, Queen's University, Kingston, Ontario, Canada, Tel: 1-613533-2727, Fax: 1-613-533-2022; E-mail: s.shafique@queensu.ca

Citation: Shafique S (2018) Neurodevelopmental Disorders and Autism in Valproic Acid Animal Models. Int J Pediatr Neonat Care 4: 146. doi: https://doi. org/10.15344/2455-2364/2018/146

Copyright: (c) 2018 Shafique. This is an open-access article distributed under the terms of the Creative Commons Attribution License, which permits unrestricted use, distribution, and reproduction in any medium, provided the original author and source are credited. 
Citation: Shafique S (2018) Neurodevelopmental Disorders and Autism in Valproic Acid Animal Models. Int J Pediatr Neonat Care 4: 144. doi: https:/doi. org/10.15344/2455-2364/2018/144

Page 2 of 4

defects, thus looking for the prevention and treatment strategies too. All the teratogens causing neural tube structural defects result in the perturbation of the fine balance of proliferation and cell death. The teratogens like VPA where apoptosis predominates results in failure of closure or fusion of the neural ridges.

There is an intimate relationship between teratogens, genetics and epigenetics. Where genetics involves the gene regulation, the epigenetics is defined as "the study of the mechanisms of temporal and spatial control of gene activity during the development of complex organisms."[8]. Xenobiotics result in the neural cell damage through various mechanisms including reactive oxygen species, DNA doublestrand breaks, defective DNA damage repair mechanisms, production of DNA adducts and epigenetic changes by histone and epigenetic code perturbations. Although these are the mechanisms of actions at the level of almost at any organ, the neuronal cell damage is no exception. The vulnerability of developing neural tissue comes from very organized cell proliferation, migration and cell death at the same time.

\section{Autism Spectrum Disorder (ASD)}

Autism spectrum disorder (ASD) is the functional neurodevelopmental condition identified by impaired social communication and repetitive/stereotyped behaviors. Interestingly, ASD pathology involves early/accelerated brain growth with large brain size and hyper-reactivity indicated by aggressive behaviors. But, at the same time involves hypo-functionality of the brain and inhibitory or negative behaviors too. During neural tissue development, the prefrontal and temporal association cortices have a slow pattern of maturation and are slightly under-developed at the time of birth [9]. The communication among neurons is of two types, within one region such as visual and auditory cortical areas, and among these regions. Normally, neurons communicate to each other in one area of brain while the communication among these brain sub-regions is in groups, while in ASD, the neurons are abnormally intense in communication with each other and the areas of other brain regions are not appropriately defined or delimited in firing For example,
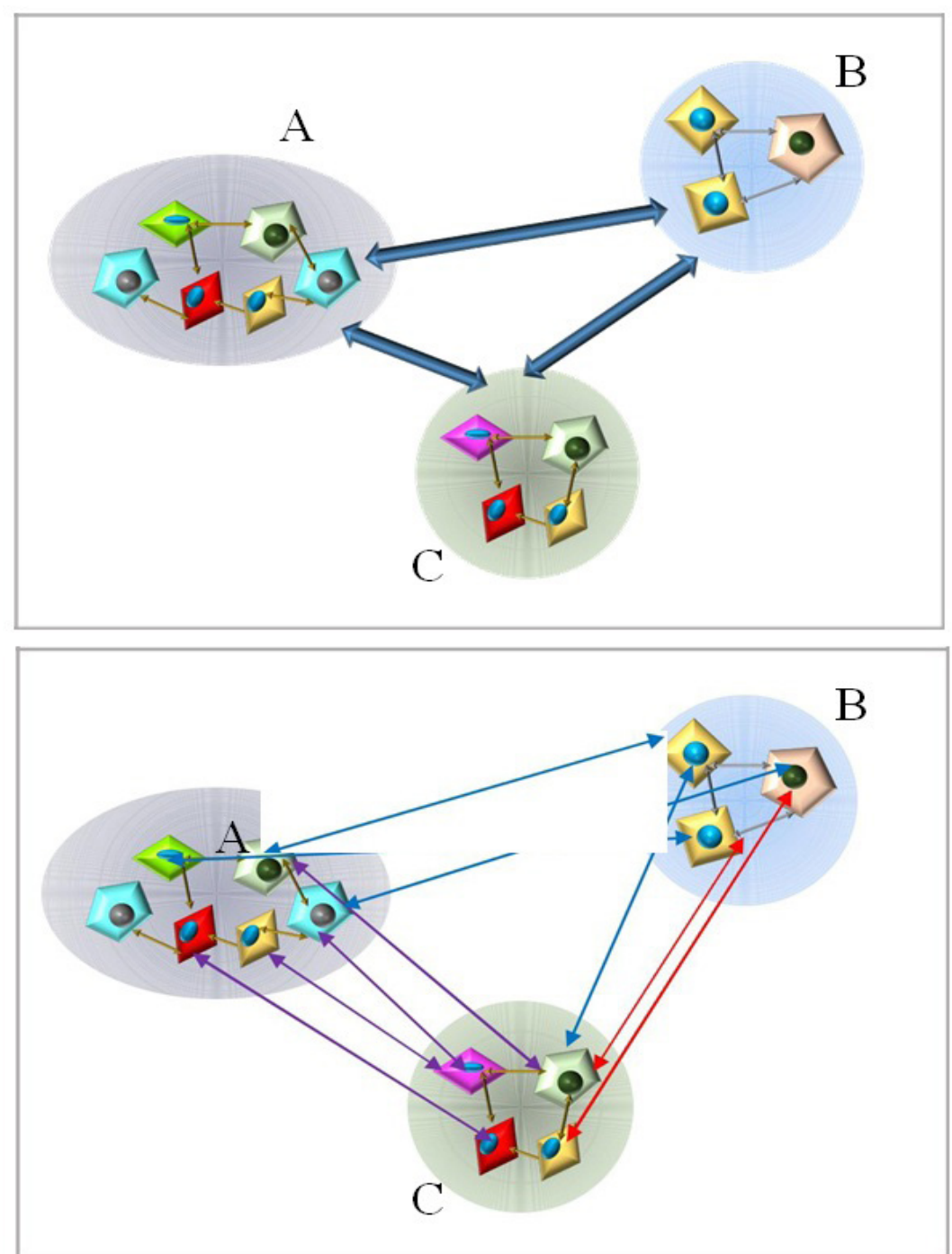

Figure 1: Areas A, B and C are groups of neurons representative of visual, Auditory and cerebellar regions. Upper panel: normal communication pattern among groups of neurons in different brain regions. Lower panel, communication pattern in autism spectrum disorder among the neurons of $\mathrm{A}, \mathrm{B}$ and $\mathrm{C}$ regions. 
visual, auditory and cerebellar regions [10]. The therapeutic options for ASD are psychological, physical and institutional-based therapies.

\section{Valproic Acid [VPA] and Autism Spectrum Disorder [ASD]}

Although there are many environmental toxicants responsible for ASD, the valproic acid is the one used as the therapeutic model to understand ASD underlying pathology at molecular and morphological levels. Understanding how VPA causes ASD is, in fact, understanding how the ASD develops and ultimately could be prevented and treated.

Evidence indicates a distinct association between VPA and ASD. The first public health prospective study on the children of women taking VPA during the pregnancy spanned over 11-years duration, showed a significant increase in neurodevelopmental disorders out of those autism being the most frequent [11]. Christensen et al. have similarly reported in 2013 of the significantly increased risk of ASD in VPAexposed children with two sub-groups, Autism Spectrum Disorder and Childhood Autism [1]. Autism Spectrum Disorder includes the manifestation of antisocial behaviors, communication difficulties and stereotyped interests [10]. Childhood Autism, on the other hand, is defined by the presence of one or more of the above symptoms during the first three years of a child [1]. The pre-school children have developmental defects in spoken language, social interactions, strong likes and dislikes as well as repetitive movements [12]. The earliest observation was observed in 1993 by Laegreid et al. of autistic-like behaviors in the children with Fetal Valproate Syndrome [13]. Thus, the animal model of ASD studies at the cellular and molecular level was developed with prenatal and postnatal VPA exposure with the construct, face and predictive validity $[14,15]$.

According to data reported by Epilepsy Society, of the 475 women of the reproductive age women on VPA were unaware of the risk for the baby of developmental disabilities in case of pregnancy [16]. The Medicines and Healthcare Products Regulatory Agency (MHRA) of the UK informs that three to four out of ten in utero exposed children would be at risk of neurodevelopmental delays and low intelligence [17]. VPA is one of the many environmental toxins having structural and functional neural tube defects, neurodevelopmental disorders and developmental delays. The awareness needs to be generated by not only prenatal counseling but also by the way as Epilepsy Society has suggested. Epilepsy Society, said, "This is a very simple and straightforward ask of the government a yearly conversation with women and girls who are taking sodium valproate, to make absolutely certain they are aware of the risks associated with the drug during pregnancy." [16].

VPA accumulates in the neuroepithelium, causes apoptosis in scattered bands resulting in the failure of anterior neuropore closure overt neural tube defects caused by VPA to include exencephaly in the developing GD9/10 mouse embryos $[18,19]$. While at the structural level, VPA interferes with cortical development inducing hypo and hyper-connectivity via affecting dendritic spine density in the forebrain [20]. In the VPA-exposed pregnant rat model, the brain of the offsprings showed reduced cerebellar volume (31\%), the decrease in the cerebellar Purkinje cells (11\%) and reduction in the number of inferior olive neurons that provide the input to the Purkinjie cells (9\%) [21].

VPA is a histone deacetylase (HDAC) inhibitor and this inhibition is most strongly associated with fetal teratogenicity. HDACs are responsible for restricted gene expression by removing the acetyl group from histone tails and restricting the transcription factors access to the promoter sites. The inhibition of these enzymes results in over-expression of genes including the proliferation of neuronal cells $[22,23]$. VPA exposure results in the accelerated dendritic patterns, neurite growth and neural proliferation through the HDAC inhibition mechanism of action. These are the similar structural changes as of observed in ASD cortices and proposed underlying mechanism of brain hyper-excitability in ASD. Therefore, VPA, after prenatal and postnatal exposure, produces the structural brain and behavioral changes similar to ASD. This not only associates the VPA as a causative agent of ASD but on the other hand making the VPAexposed rodents, rats and mice, an ideal model to study ASD [24].

\section{Conclusion}

ASD and related learning disabilities are associated with the developmental origins of health and diseases (DOHAD), a group consisting of the diseases affecting the future human generations. Awareness and future research on the VPA-exposed pre and postnatal rodent models of ASD may identify the underlying pathology and therapeutic options. One example is current treatment of ASD with intranasal oxytocin in rat model [25]. The similarities between the identified structural brain pathologies in the ASD affected brains and VPA-exposed brains provide the investigation of molecular mechanisms for ASD development on VPA-exposed mouse and rat models. The treatment options for overt birth defects, where treatable as spina bifida, can be planned and started at the time of birth. On the contrary, the functional defects are not even diagnosed till a certain age of the postnatal growth. The unfortunate part is that the problems remain with the individuals for the rest of their lives. Therefore, research in this domain is the need of the hour.

\section{Competing Interests}

The author declare that no competing interests is present.

\section{References}

1. Christensen J, Grønborg TK, Sørensen MJ, Schendel D, Parner ET et al. (2013) Prenatal valproate exposure and risk of autism spectrum disorders and childhood autism. JAMA 309: 1696-1703.

2. Neural Tube Defects: Condition Information (2017) National Institute of Child Health and Human Development. U.S. National Institutes of Health.

3. Lozano R, Naghavi M, Foreman K, Lim S, Shibuya K, et al. (2012) Global and regional mortality from 235 causes of death for 20 age groups in 1990 and 2010: a systematic analysis for the Global Burden of Disease Study 2010. Lancet. 380: 2095-128.

4. America's Children and the Environment.

5. Samaco RC, Hogart A, LaSalle JM (2005) Epigenetic overlap in autismspectrum neurodevelopmental disorders: MECP2 deficiency causes reduced expression of UBE3A and GABRB3. Hum Mol Genet 14: 483-492.

6. Milunsky A, Jick H, Jick SS, Bruell CL, MacLaughlin DS, et al. (1989) Multivitamin/folic acid supplementation in early pregnancy reduces the prevalence of neural tube defects. JAMA 262: 2847-2852.

7. Copp AJ, Greene NDE (2010) Genetics and development of neural tube defects. J Pathol 220: 217-230.

8. Holliday R (1990) DNA Methylation and Epigenetic Inheritance. Philos Trans R Soc Lond B Biol Sci 326: 329-338.

9. Gogtay N, Giedd JN, Lusk L, Hayashi KM, Greenstein D et al. (2004) Dynamic mapping of human cortical development during childhood through early adulthood. Proceedings of the National Academy of Sciences of the United States of America 101: 8174-8179.

10. Belmonte MK, Allen G, Beckel-Mitchener A, Boulanger LM, Carper RA, et al. (2004) Autism and abnormal development of brain connectivity. J Neurosci 24: 9228-9231. 
Citation: Shafique S (2018) Neurodevelopmental Disorders and Autism in Valproic Acid Animal Models. Int J Pediatr Neonat Care 4: 144. doi: https://doi. org/10.15344/2455-2364/2018/144

Page 4 of 4

11. Bromley RL, Mawer GE, Briggs M, Cheyne C, Clayton-Smith J, et al. (2013) The prevalence of neurodevelopmental disorders in children prenatally exposed to antiepileptic drugs. J Neurol Neurosurg Psychiatry 84: 637-643.

12. American Psychiatric Association (2000) Diagnosticand Statistical Manual of Mental Disorders. Text Revision. Washington, DC: American Psychiatric Association.

13. Laegreid L, Kyllerman M, Hedner T, Hagberg B, Viggedahl G, et al. (1993) Benzodiazepine amplification of valproate teratogenic effects in children of mothers with absence epilepsy. Neuropediatrics 24: 88-92.

14. Schneider T, Turczak J, Przewłocki R (2006) Environmental enrichment reverses behavioral alterations in rats prenatally exposed to valproic acid: issues for a therapeutic approach in autism. Neuropsychopharmacology 31 36-46

15. Roullet FI, Lai JK, Foster JA (2013) In Utero exposure to valproic acid and autism-a current review of clinical and animal studies. Neurotoxicol Teratol 36: 47-56.

16. Wise J (2017) Women still not being told about pregnancy risks of valproate Bmj.

17. Medicines and Healthcare products Regulatory Agency.

18. Kultima K, Nyström AM, Scholz B, Gustafson AL, Dencker L, et al. (2004) Valproic acid teratogenicity: A toxicogenomics approach. Environ Health Perspect 112: 1225-1235.

19. Dencker L, Nau H, D'Argy R (1990) Marked accumulation of valproic acid in embryonic neuroepithelium of the mouse during early organogenesis. Teratology 41: 699-706.

20. Bringas ME, Carvajal-Flores FN, López-Ramírez TA, Atzori M, Flores G, et al. (2013) Rearrangement of the dendritic morphology in limbic regions and altered exploratory behavior in a rat model of autism spectrum disorder. Neuroscience 241: 170-187

21. Ingram JL, Peckham SM, Tisdale B, Rodier PM (2000) Prenatal exposure of rats to valproic acid reproduces the cerebellar anomalies associated with autism. Neurotoxicol Teratol 22: 319-324.

22. Lloyd KA (2013) A scientific review: mechanisms of valproate-mediated teratogenesis. Bioscience Horizons 6: hzt003-hzt003.

23. Göttlicher $M$, Minucci $S$, Zhu $P$, Krämer $\mathrm{OH}$, Schimpf $A$, Giavara $S$, et al (2001) Valproic acid defines a novel class of HDAC inhibitors inducing differentiation of transformed cells. EMBO J 20: 6969-6978.

24. Go HS, Kim KC, Choi CS, Jeon SJ, Kwon KJ, et al. (2012) Prenatal exposure to valproic acid increases the neural progenitor cell pool and induces macrocephaly in rat brain via a mechanism involving the GSK-3beta/betacatenin pathway. Neuropharmacology 63: 1028-1041.

25. Dai YC, Zhang HF, Schön M, Böckers TM, Han SP, et al. (2018). Neonatal Oxytocin Treatment Ameliorates Autistic-Like Behaviors and Oxytocin Deficiency in Valproic Acid-Induced Rat Model of Autism. Front Cell Neurosci 12: 355. 